\title{
Improved eukaryotic promoter-detection vector carrying two luciferase reporter genes
}

\author{
Madhu S. Malo, Mario Abedrapo, Alex Chen, Moushumi Mozumder, \\ Premraj Pushpakaran, Fuad Alkhoury, Wenying Zhang, Elizabeth Fleming, \\ and Richard A. Hodin \\ Harvard Medical School, Boston, MA, USA
}

BioTechniques 35:1150-1154 (December 2003)

Understanding gene regulation is of fundamental importance to virtually all biological systems. A gene is regulated through cis-acting regulatory DNA sequences (elements) in conjunction with trans-acting regulatory proteins. The cis-acting regulatory elements involved in transcription include, for example, the promoter, enhancer, silencer, specific activator binding sites, and specific repressor binding sites, whereas the trans-acting regulatory proteins include basic transcription factors, activators, repressors, coactivators, and co-

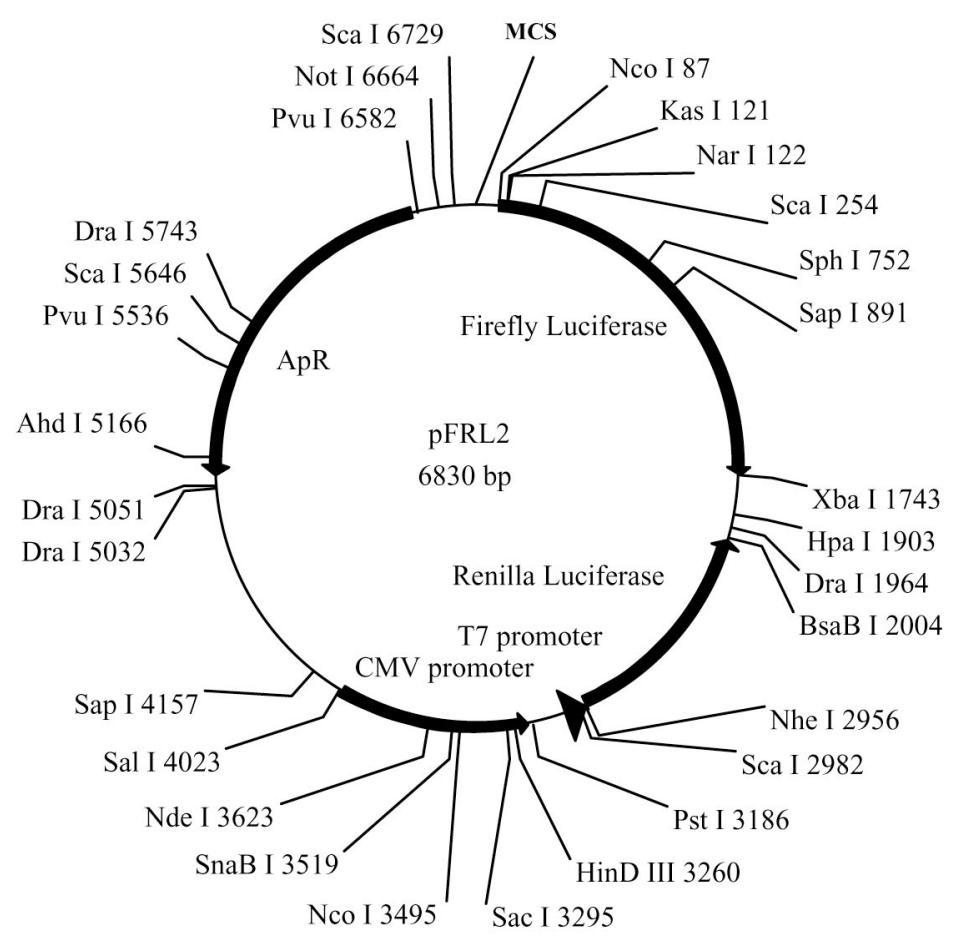

MCS: GGTACCGAGCTCTTACGCGTGCTAGCCCGGGCTCGAGATCTGCGATCTAAGTAAGCTT

Kpn I Sac I Mlu I Nhe I Xma I Sma I Xho I Bgl II

Figure 1. Structure of the eukaryotic promoter-detection vector pFRL2. pFRL1 (not shown) was constructed by cloning the 2.3-kb BglII-BamHI fragment carrying the Renilla luciferase gene from pRLCMV into the pGL3-Basic vector so that both genes were in the same orientation. This strategy could not produce clones with genes in opposite orientations, probably due to instability from duplication of the same long poly(A) signal after the firefly and Renilla luciferase genes in pRL-CMV and pGL3-Basic. pFRL2 (shown) was constructed by cloning the 2.0-kb BglII-XbaI (blunt-ended) fragment of pRL-CMV carrying the Renilla luciferase gene into pGL3-Basic digested with BamHI (blunt-ended) so that the luciferase genes would transcribe in opposite orientations (arrows). The sequence of multiple cloning sites (MCS) is shown. Arrowhead, the orientation of the T7 phage promoter. CMV, cytomegalovirus. repressors. For the quantitative analysis of transcriptional regulatory elements, a variety of reporter vectors have been developed based on firefly luciferase and Renilla luciferase, chloramphenicol acetyltransferase (CAT), $\beta$-galactosidase, $\beta$-glucuronase, $\beta$-lactamase, alkaline phosphatase, human growth hormone $(\mathrm{hGH})$, and green fluorescent protein (GFP) (1-11). Luciferase reporter vectors have become the vectors of choice for most investigations because of the availability of rapid and sensitive luciferase assay systems (1).

Most of the currently available eukaryotic promoter-detection vectors carry only a single reporter gene. The use of such a vector can generate erroneous results due to the variability in transfection efficiency (intracellular copy number) in different samples of transfected cells. Many individual factors can affect the transfection efficiency of a plasmid, including contaminating nucleases, endotoxins, and salts in DNA preparations. Dispensation of variable amounts of DNA during pipetting of small quantities of DNA can also influence transfection efficiency. The size of a plasmid also plays a role in transfection because some smaller plasmids can enter cells more efficiently. To minimize the error due to variable transfection efficiency, current protocols generally use a second control plasmid to normalize the data for the test plasmid. The normalized value of the test plasmid data is usually expressed as a percentage of the control plasmid data. However, the use of a control plasmid cannot completely eliminate variability in transcription efficiency because of contamination of individual samples and the variable quantity of individual plasmid from sample to sample. To solve this problem, which is associated with the use of two plasmids, Park (1) developed a promoter-detection vector, $\mathrm{pJDL}_{\mathrm{cmv}}$, which carries both the firefly and Renilla luciferase genes. However, the two luciferase genes in this vector transcribe in the same clockwise direction, thus possibly expressing any fragment cloned upstream of the firefly luciferase gene due to "leaky" transcription from the cytomegalovirus (CMV) promoter driving the Renilla luciferase gene. For instance, Giannakis et al. (12) have described readthrough 
transcription artifacts associated with pGL3-Basic (Promega, Madison, WI, USA) and pCAT vectors. To address this potential problem, Park (1) used a poly(A) site downstream of the $R e$ nilla luciferase gene to pause or stop transcription from the CMV promoter. However, our experience has shown that poly(A) signals do not completely stop transcription from an upstream promoter, especially in the case of a very strong promoter like CMV (unpublished observation; see Figure 2). Thus, any result obtained by using $\mathrm{pJDL}_{\mathrm{cmv}}$ could be subject to misinterpretation. To eliminate the problem associated with leaky transcription, we sought to construct a promoter-detection vector carrying the luciferase genes in opposite orientations.

We cloned the Renilla luciferase gene from pRL-CMV (Promega) into the pGL3-Basic vector to construct pFRL1, which is equivalent to $\mathrm{pJDL}_{\mathrm{cmv}}$ (1) and carries both genes in a clockwise orientation. Similarly, we constructed pFRL2, in which the Renilla and firefly luciferase genes are transcribed in opposite orientations (Figure 1). We then compared the basal level of transcription of the firefly luciferase genes in pGL3-Basic, pFRL1, and pFRL2.
We transfected Caco-2 human colon carcinoma cells with different amounts of individual vector to determine the experiment-to-experiment variation in the basal level of firefly luciferase transcription in each vector. To normalize the luciferase activity in pGL3-Basic, we cotransfected pRL-CMV. Relative firefly luciferase activities were determined after normalizing the data based on the Renilla luciferase activity for each sample (Figure 2A). As expected, the results show that the basal level of transcription for pFRL1 and pFRL2 is consistent for all concentrations of DNA; however, the level of basal transcription is much (>3.5-fold) higher for pFRL1. The results also show that the level of basal transcription is dose-dependent in pGL3-Basic, presumably due to variation in the intracellular copy number. These results suggest that pFRL2 would be the best among these three vectors for eukaryotic promoter analysis.

To evaluate the efficacy of the promoter-detection vectors pGL3-Basic, pFRL1, and pFRL2, we cloned the proximal $2.5-\mathrm{kb}$ promoter region of the human intestinal alkaline phosphatase $(I A P)$ gene, an enterocyte differentiation marker, into these vectors, thus generating pIAP-2574/-49, pFRL1-IAP1, and
pFRL2-IAP1, respectively. The IAP gene is activated by thyroid hormone (T3) (13). T3 mediates its action through its nuclear receptor, which binds as a transcription factor to DNA cis-elements named thyroid hormone-response elements (TREs). For various amounts of transfected reporter plamid, $\mathrm{T} 3$ activation was determined by finding the ratio of the relative firefly luciferase activities with and without T3 (Figure 2B). The results show that the activation ratio in the case of pIAP2574/-49, the derivative of pGL3-Basic, varies from 32- to 67 -fold, thus rendering the interpretation of results very difficult. However, the activation ratio is consistent at approximately 15 - and 6-fold in the cases of pFRL2-IAP1 and pFRL1IAP1, respectively. The lower activation ratio in pFRL1-IAP1 is mostly due to an increased basal level of transcription in pFRL1-IAP1, which is approximately 4-fold higher (data not shown) compared to that of pFRL2-IAP1. This result is consistent with the higher level of basal transcription of firefly luciferase associated with the pFRL1 vector. T3-induced activation of the endogenous IAP gene as determined by Northern blot analysis (data not shown) also approximates the values achieved by transfection assays using pFRL2-IAP1 and pFRL1-IAP1.
A

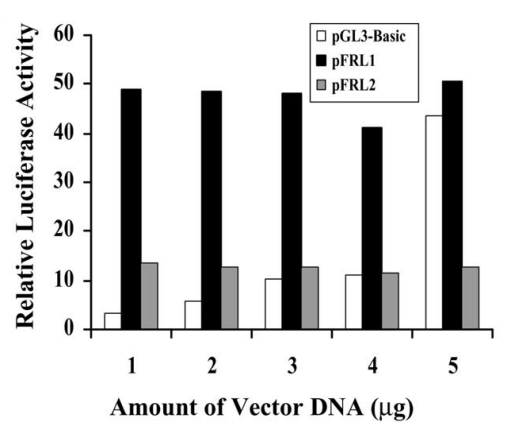

B

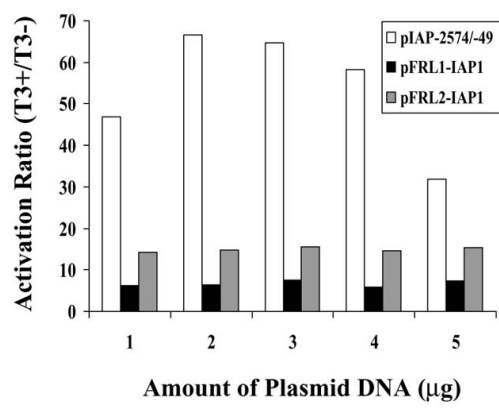

C

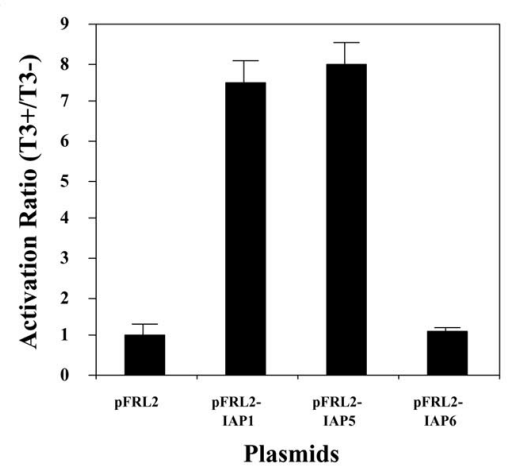

Figure 2. Transfection assays using the eukaryotic promoter-detection vector pFRL2. Caco-2 cells were transiently transfected with the indicated amount of vector or plasmid DNA. pRL-CMV $(0.5 \mu \mathrm{g})$ was added to each transfection sample containing pGL3-Basic or its derivatives. The amount of total DNA in each reaction was kept the same $(7.5 \mu \mathrm{g})$ using nonspecific plasmid DNA. Relative firefly luciferase activity was determined as the percentage of the Renilla luciferase activity. (A) Individual experimental variability of basal transcription level of the firefly luciferase gene in pGL3-Basic, pFRL1, and pFRL2. (B) Individual experimental variability of T3-induced transcriptional activation of the intestinal alkaline phosphatase (IAP) gene in pIAP-2574/-49, pFLR1-IAP1, and pFRL2-IAP1, the derivatives of pGL3-Basic, pFRL1, and pFRL2, respectively, carrying the IAP proximal promoter region between -2574 and - 49 in relation to the ATG start codon. The cells were also cotransfected with a plasmid expressing the thyroid hormone receptor TR $\beta 1(1.5 \mu \mathrm{g})$ and treated with or without T3 (100 nM). (C) T3-induced transcriptional activation of the IAP gene in different $5^{\prime}$ deletion mutations of pFRL2-IAP1. The plasmid pFRL2-IAP5 was constructed by deleting a 1.83-kb SacIPflMI restriction fragment from pFRL2-IAP1. Similarly, pFRL2-IAP6 was constructed by deleting a 1.96-kb SacI-BstEII restriction fragment from pFRL2-IAP1. During transient transfection analysis, the cells were also cotransfected with a plasmid expressing the thyroid hormone receptor TR $\alpha 1(1.5 \mu \mathrm{g})$ and treated with or without T3 $(100 \mathrm{nM})$. The data indicate the mean $(\bar{x} \pm \mathrm{SD})$ of three independent experiments. 
Thus, it appears that the use of a single dual-luciferase reporter vector yields more reliable results than normalization via separate vectors. Furthermore, the lower basal transcription of the firefly luciferase gene in pFRL2 makes it more suitable than $\mathrm{pFRL} 1$ for promoter analysis. Even so, the existence of extra regulatory elements of the CMV promoter in pFRL2 may unduly influence the activity of a weak test promoter by squelching the basic transcription machinery or even by competing for sequence specific transcription factors. In addition, the vector may not be suitable for studying the effects of any activator or repressor that affects the activity of both the test promoter and the CMV promoter.

To test the efficacy of pFRL2 for promoter analysis, we then evaluated derivatives of pFRL2 carrying different $5^{\prime}$ deletions of the IAP promoter to localize the TRE in the IAP promoter. We deleted $1.83 \mathrm{~kb}$ from the $5^{\prime}$ end of the IAP promoter to construct pFRL2IAP5, which carries the IAP promoter region between -750 and -49 in relation to the ATG start codon. A larger deletion created pFRL2-IAP6, which carries the -616 to -49 region of the IAP promoter. We separately transfected Caco- 2 cells with these plasmids, measured the firefly luciferase activity for each sample, normalized based on the Renilla luciferase activity, and determined the activation ratio (T3+/T3-) (Figure 2C). The data show that T3induced activation is lost in the case of pFRL2-IAP6, thus indicating that the TRE in the IAP promoter is localized in the 135-bp region between -750 and -616. Using electrophoretic mobility shift assay and in vitro site-directed mutagenesis, we also confirmed the existence of a functional TRE between -632 and -612 (data not shown). We conclude that pFRL2 is a very useful vector for studying eukaryotic promoters, reducing the problems of copy number variation and leaky transcription that are associated with other eukaryotic promoter-detection vectors.

\section{ACKNOWLEDGMENTS}

This work was supported by National Institutes of Health research grant nos. DK47186 and DK50623 to R.A.H.

\section{REFERENCES}

1.Park, J.B. 2001. Concurrent measurement of promoter activity and transfection efficiency using a new reporter vector containing both Photinus pyralis and Renilla reniformis luciferase genes. Anal. Biochem. 291:162-166.

2.Prost, E. and D.D. Moore. 1986. CAT vectors for analysis of eukaryotic promoters and enhancers. Gene 45:107-111.

3.Macgregor, G.R. and G.P. Nolan. 1991. Gene transfer and expression protocols, $\mathrm{p}$ 217-235. In E.J. Murray and J.M. Walker (Eds.), Humana Press, Clifton, NJ.

4.Mitchell, P.J. and R. Tjian. 1989. Transcriptional regulation in mammalian cells by sequence-specific DNA binding proteins. Science 245:371-378.

5.Nguyen, V.T., M. Morange, and O. Bensaude. 1988. Firefly luciferase luminescence assays using scintillation counters for quantitation in transfected mammalian cells. Anal Biochem. 171:404-408.

6.Gorman, C.M., L.F. Moffat, and B.H. Howard. 1982. Recombinant genomes, which express chloramphenicol acetyltransferase in mammalian cells. Mol. Cell Biol. 2:10441051.

7.Alam, J. and J.L. Cook. 1990. Reporter genes: application to the study of mammalian gene transcription. Anal. Biochem. 188: 245-254.

8.Berger, J., J. Hauber, R. Hauber, R. Geiger, and B.R. Cullen. 1988. Secreted placental alkaline phosphatase: a powerful new quantitative indicator of gene expression in eukaryotic cells. Gene 15:1-10.

9.Chalfie, M., Y. Tu, G. Euskirchen, W.W. Ward, and D.C. Prasher. 1994. Green fluorescent protein as a marker for gene expression. Science 263:802-805

10.Katz, R.W. and R.J. Koenig. 1994. Specificity and mechanism of thyroid hormone induction from an octamer response element. J. Biol. Chem. 269:18915-18920.

11.Koenig, R.J., R.L. Warne, G.A. Brent, J.W. Harney, P.R. Larsen, and D.D. Moore. 1988. Isolation of a cDNA clone encoding a biologically active thyroid hormone receptor. Proc. Natl. Acad. Sci. USA 85:5031-5035.

12.Giannakis, G., S.R. Edmondson, J.M. Favaloro, J.D. Zajac, and K.J. Greenland. 2003. Aberrant cryptic responsiveness of the pCAT 3- and pGL3-promoter reporter vectors. BioTechniques. 35:332-339.

13.Hodin, R.A., A. Shei, M. Morin, and S. Meng. 1996. Thyroid hormone and the gut: selective transcriptional activation of a villusenterocyte marker. Surgery 120:138-143.

Received 17 September 2003; accepted 3 October 2003.

Address correspondence to Richard A. Hodin, Department of Surgery, Massachusetts General Hospital, Gray 504, 55 Fruit Street, Boston, MA 02114, USA. e-mail: rhodin@partners.org 